\title{
General procedure for solution of contact problems under dynamic normal and tangential loading based on the known solution of normal contact problem
}

\author{
Valentin L Popov, Roman Pohrt and Markus Heß
}

\begin{abstract}
In this article, we show that the normal contact problem between two elastic bodies in the half-space approximation can always be transformed to an equivalent problem of the indentation of a profile into an elastic Winkler foundation. Once determined, the equivalent profile can also be used for tangential contact problems and arbitrary superimposed normal and tangential loading histories as well as for treating of contact problems with linearly viscoelastic bodies. In the case of axis-symmetric shapes, the equivalent profile is given by the method of dimensionality reduction integral transformation. For all other shapes, the profile is deduced from the solution of the elastic contact normal problem, which can be obtained numerically or experimentally.
\end{abstract}

\author{
Keywords \\ Friction, sliding, contact, method of dimensionality reduction, Mindlin
}

Date received: 31 July 20I5; accepted: I5 January 2016

\section{Introduction}

Contact mechanics and friction play a key role in many technological and biological systems. Due to the multiscale roughness of the contacting surfaces, the treatment of the associated contact problems proves to be difficult. Even the simple case of a non-adhesive, frictionless normal contact problem between two linear elastic solids with randomly rough surfaces is still a controversial scientific issue. Several analytical and numerical methods were developed to deal with the normal contact problem. Usually, numerical calculations are based on finite element method, ${ }^{1}$ boundary element method $^{2}$ or Green's function molecular dynamics, ${ }^{3}$ each of which has certain advantages over the other methods. A broad overview with discussion of the existing numerical and analytical methods can be found in Yastrebov et al. ${ }^{4}$

It is self-explanatory that the presence of friction makes the contact problem more complicated. In the classical uncoupled tangential contact problem between two linear elastic spheres, Cattaneo ${ }^{5}$ and Mindlin ${ }^{6}$ assumed a constant normal force $F_{N}$ and a subsequently applied, increasing tangential force $F_{x}$. It is well-known that this kind of loading results in the formation of a slip domain near the boundary of the contact area, while the inner domain remains in stick. However, this tangential contact problem becomes more complex for arbitrary loading scenarios since the state of stress depends not only upon the initial state of loading but also upon the complete loading history. ${ }^{7}$

One could assume that considering the tangential contact of nominally flat rough surfaces under arbitrary varying loads will increase the difficulty of the contact problem even further. However, this is not the case, due to the Ciavarella-Jäger theorem. Jäger ${ }^{8,9}$ and Ciavarella ${ }^{10,11}$ independently showed that the tangential stresses in the tangential contact problem are equivalent to the difference between the actual normal stresses and those that correspond to a smaller contact area (the stick area), both multiplied with the

Institut für Mechanik, Technische Universität Berlin, Berlin, Germany

\section{Corresponding author:}

Roman Pohrt, Institut für Mechanik, Technische Universität Berlin, Sek. C8-4, Straße des 17 Juni I35, 10623 Berlin, Germany.

Email: roman.pohrt@tu-berlin.de 
coefficient of friction. The Ciavarella-Jäger theorem holds for all two-dimensional contact problems between solids of elastic similar materials irrespective of whether the contact area is simply connected or even spread over multiple spots. For all three-dimensional (3D) contact problems of elastic similar bodies including the classical problem of Cattaneo and Mindlin, it only applies in an approximate sense. The classical problem states that the frictional stresses in the slip domain are all directed in the direction of the applied tangential force. With the exception of the unrealistic case of $v_{1}=v_{2}=0$, this assumption violates the condition that at every point in the slip domain, the slip opposes the direction of tangential stress. The reason for this is the presence of an additional deformation perpendicular to the direction of the applied force. For the classical contact of parabolic bodies, however, it could be proven that this component may be neglected. ${ }^{12,13} \mathrm{We}$ assume that this approximation is also valid for the generalization of the CattaneoMindlin theory for arbitrary contacts including contact between bodies with randomly rough surfaces. For the latter case, a series of articles ${ }^{14-16}$ investigated the frictional energy dissipation generated by varying normal and tangential forces by use of the theorem.

A further immediate consequence of the CiavarellaJäger theorem is the possibility of replacing the contact problem of an approximately isotropic surface shape by an equivalent axis-symmetric contact problem. Provided that the elastic normal contact problem has been solved, the equivalent profile can be deduced starting from the Galin-Sneddon integral equation. ${ }^{17,18}$ Aleshin et al. ${ }^{19}$ followed this way and studied the tangential contact of the equivalent axis-symmetric profile for arbitrary loading scenarios by the method of memory diagrams (MMDs). In contrast to the work of Mindlin and Deresiewicz, ${ }^{7}$ the MMD replaces the complex traction distribution inside the contact area by a simple internal function containing the same memory information. Therefore, the MMD is a powerful tool to calculate the hysteretic tangential force-displacement curves resulting from an arbitrary loading scenario of frictional contact problems. In 2006, Borri-Brunetto et al. ${ }^{20}$ used the Ciavarella-Jäger theorem to show how the MindlinDeresiewicz procedure can be generalized for cases when the tangential load is reversed. After each reversing of the force direction, they give the dependencies of force and displacement. Using this approach, the energy dissipation for oscillating load is calculated.

For the case of axis-symmetric profiles, the method of dimensionality reduction (MDR) $)^{21-23}$ is an elegant and powerful procedure for evaluating both normal and tangential contact. It starts by generating a onedimensional (1D) profile which corresponds to the axis-symmetric shape. Following the ideas of Lee and Radok, ${ }^{24}$ the MDR can also be used for solving normal contact problems that involve linear viscoelastic media.

We thus know that for every arbitrary 3D contact, there is an equivalent axis-symmetric problem and that for any axis-symmetric shape there is an equivalent 1D profile.

In this article, we will show a generalized rule for obtaining the equivalent $1 \mathrm{D}$ profile which only depends on the original 3D geometry. Once the 1D equivalent profile is found, the numerical procedures of MDR can be applied for both normal and tangential contact. These procedures consist of only linear equations with independent degrees of freedom. In the first section, we will show how a general $1 \mathrm{D}$ profile is obtained from a known solution of the frictionless elastic indentation problem. In the following section, it will be displayed why and how this equivalent profile can be used in order to simulate the dynamic tangential contact. The principle used is closely related to the procedure of Borri-Brunetto et al. ${ }^{20} \mathrm{We}$ will then focus on how to obtain the equivalent profile for different geometries. Finally, we will show some numerical examples.

\section{Equivalent elastic foundation and equivalent profile}

Consider a contact between an elastic indenter of arbitrary shape $\mathrm{z}=\mathrm{f}(\mathrm{x}, \mathrm{y})$ with an elastic half-space. From the contacting bodies' Young's moduli $E_{1}$ and $E_{2}$, Poisson's ratios $\nu_{1}$ and $\nu_{2}$ and moduli of shear $G_{1}$ and $\mathrm{G}_{2}$, we define the reduced moduli

$$
\begin{aligned}
& \mathrm{E}^{*}=\left(\frac{1-v_{1}^{2}}{\mathrm{E}_{1}}+\frac{1-v_{2}^{2}}{\mathrm{E}_{2}}\right)^{-1} \\
& \text { and } \mathrm{G}^{*}=\left(\frac{2-v_{1}}{4 \mathrm{G}_{1}}+\frac{2-v_{2}}{4 \mathrm{G}_{2}}\right)^{-1}
\end{aligned}
$$

During the indentation, the normal force $\mathrm{F}_{\mathrm{N}}$ is a continuous, monotonically increasing function of the indentation depth $\mathrm{d}$. Therefore, we can define unambiguously an incremental stiffness

$$
\mathrm{k}=\frac{\mathrm{dF}_{\mathrm{N}}}{\mathrm{dd}}
$$

which can also be expressed as a unique function of the indentation depth

$$
\mathrm{k}=\mathrm{k}(\mathrm{d})
$$

Let us introduce formally the "contact length," sometimes called "Holm radius" in the literature

$$
1=\frac{k}{2 \mathrm{E}^{*}}
$$

The indentation depth is a unique function of the contact stiffness and thus of the contact length 1

$$
\mathrm{d}=\mathrm{g}(1)
$$

Note that 1 has the unit length and depends only on the topography and the indentation depth (also in unit length). Equation (5) thus links only geometrical quantities, independently on the material properties. 
Let us consider the process of indentation from its very first moment until the final indentation depth $d$, the current values of the normal force and indentation depth being given by $\tilde{\mathrm{F}}_{\mathrm{N}}, \tilde{\mathrm{d}}$. During the indentation, the indentation depth changes from $\tilde{\mathrm{d}}=0$ to $\tilde{\mathrm{d}}=\mathrm{d}$, the normal force changes from $\tilde{\mathrm{F}}_{\mathrm{N}}=0$ to $\tilde{\mathrm{F}}_{\mathrm{N}}=\mathrm{F}_{\mathrm{N}}$ and the contact length from $\overline{1}=0$ to $\bar{l}=1$. The final normal force can be written as

$$
\begin{aligned}
\mathrm{F}_{\mathrm{N}} & =\int_{0}^{\mathrm{F}_{\mathrm{N}}} \mathrm{d} \tilde{\mathrm{F}}_{\mathrm{N}}=\int_{0}^{1} \frac{\mathrm{d} \tilde{\mathrm{F}}_{\mathrm{N}}}{\mathrm{d} \tilde{\mathrm{d}}} \frac{\mathrm{d} \tilde{\mathrm{d}}}{\mathrm{d} \overline{\mathrm{l}}} \mathrm{d} \overline{\mathrm{l}} \\
& =\int_{0}^{1} \overline{\mathrm{k}} \frac{\mathrm{d} \tilde{\mathrm{d}}}{\mathrm{d} \overline{\mathrm{l}}} \mathrm{d} \overline{\mathrm{l}}=2 \mathrm{E}^{*} \int_{0}^{1} \overline{\mathrm{l}} \frac{\mathrm{dg}(\overline{\mathrm{l}})}{\mathrm{d} \overline{\mathrm{l}}} \mathrm{d} \overline{\mathrm{l}}
\end{aligned}
$$

which gives after partial integration

$$
\mathrm{F}_{\mathrm{N}}=2 \mathrm{E}^{*}\left[1 \cdot g(\mathrm{l})-\int_{0}^{1} \mathrm{~g}(\overline{\mathrm{l}}) \mathrm{d} \overline{\mathrm{l}}\right]=2 \mathrm{E}^{*} \int_{0}^{1}(\mathrm{~d}-\mathrm{g}(\overline{\mathrm{l}})) \mathrm{d} \overline{\mathrm{l}}
$$

This equation can be easily interpreted as a force resulting from the indentation of the profile (5) into an elastic foundation as defined by the MDR. ${ }^{22}$

Indeed, consider an elastic foundation of independent springs with equal distance $\Delta \mathrm{x}$, each having the normal stiffness

$$
\Delta \mathrm{k}_{\mathrm{z}}=\mathrm{E}^{*} \Delta \mathrm{x}
$$

as depicted in Figure 1. The tangential stiffness of each spring is given by

$$
\Delta \mathrm{k}_{\mathrm{x}}=\mathrm{G}^{*} \Delta \mathrm{x}
$$

If the profile $\mathrm{g}(\mathrm{x})$ is pressed into the elastic foundation defined by equation (8), the surface displacement in the normal direction at any point $\mathrm{x}$ will be given by the difference of the indentation depth $\mathrm{d}$ and the profile shape $\mathrm{g}(\mathrm{x})$

$$
\mathrm{u}_{\mathrm{z}}^{1 \mathrm{D}}(\mathrm{x})=\mathrm{d}-\mathrm{g}(\mathrm{x})
$$

For contacts without adhesion, the displacement vanishes at the edge of the contact

$$
\mathrm{u}_{\mathrm{z}}^{1 \mathrm{D}}(\mathrm{l})=\mathrm{d}-\mathrm{g}(1)=0
$$

The normal force in a single spring is given by

$$
\Delta \mathrm{F}_{\mathrm{N}}(\mathrm{x})=\Delta \mathrm{k}_{\mathrm{z}}(\mathrm{d}-\mathrm{g}(\mathrm{x}))=\mathrm{E}^{*}(\mathrm{~d}-\mathrm{g}(\mathrm{x})) \Delta \mathrm{x}
$$

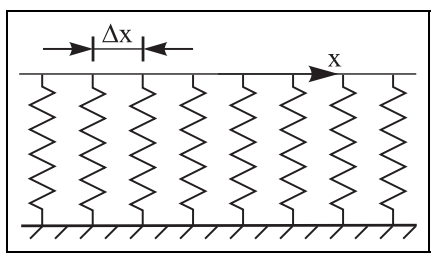

Figure I. Equivalent elastic foundation. from which the total normal force in the equilibrium state can be calculated by summation over all springs. In the limiting case $\Delta \mathrm{x} \rightarrow 0$, the sum will be the integral

$$
F_{N}=E^{*} \int_{-1}^{1} u_{z}^{1 D}(x) d x=2 E^{*} \int_{0}^{1}(d-g(x)) d x
$$

It can be seen easily that equations (11) and (13) reproduce equations (5) and (7). Therefore, the profile $\mathrm{g}(\mathrm{x})$ is the geometrical interpretation of the dependence $\mathrm{d}=\mathrm{g}(\mathrm{l})$ for the given 3D profile shape.

In order to generate the equivalent profile for a given 3D topography, three different procedures are at our disposal.

When the original indenting shape is an axissymmetric profile $f(r)$ which depends only the radial coordinate $r$ and has a compact (circular) contact area, then the equivalent profile $\mathrm{g}(\mathrm{x})$ is given by the MDR transformation

$$
g(x)=|x| \int_{0}^{|x|} \frac{f^{\prime}(r)}{\sqrt{x^{2}-r^{2}}} d r
$$

stemming from the well-known solution of GalinSneddon for the normal contact problem of axissymmetric profiles. One can either evaluate equation (14) or compose the equivalent profile using a Taylor series of $\mathrm{f}(\mathrm{r})$. For more details, see Popov and $\mathrm{He}^{22}$ (Chapter 3).

In the case of non-axisymmetric profiles, the equivalent profile also does always exist, but the transformation rule is generally not known. In some special cases, an equivalent profile can also be found for complicated, non-axisymmetric surface geometries. This is the case when an analytical solution of the normal indentation is available. Consider for instance fractal rough surfaces with given Hurst exponent $\mathrm{H}$. It has been shown in Pohrt et al. ${ }^{25}$ that here (with some statistical deviation stemming from the randomness) the normal force depends on the indentation depth as

$$
\mathrm{F}(\mathrm{d}) \propto \mathrm{d}^{\frac{\mathrm{H}+1}{\mathrm{H}}} \quad 1 \propto \mathrm{d}^{\frac{1}{\mathrm{H}}}
$$

We can thus derive the equivalent profile in the form of $\mathrm{g}(\mathrm{x})=$ const $\cdot \mathrm{x}^{\mathrm{H}}$.

In all other cases, the dependency between the Holm radius and the indentation depth can be obtained experimentally or numerically. The boundary element method is suitable for the later and some examples of such simulations and their respective equivalent profiles can be found in section "Examples of equivalent profiles."

For the experimental approach, the dependency can be found by indenting the original shape into a soft linear elastic counterpart such as a silicon rubber and recording both the penetration depth and the resulting normal force. The derivative of the normal force 
normalized by the effective Young's modulus then gives the Holm radius.

\section{Solution of tangential contact using equivalent profiles}

As shown in Popov and $\mathrm{He} \beta,{ }^{22}$ the tangential contact can be described in the frame of the MDR by assuming for the interaction of springs in the equivalent MDR model Coulomb's law of friction with the same coefficient of friction as in the original $3 \mathrm{D}$ contact problem. That is, it is assumed that a spring sticks to the profile if the tangential force caused by the tangential displacement of the profile does not exceed the normal force acting in this spring multiplied by the coefficient of friction, and it is equal to the normal force multiplied to the coefficient of friction in the sliding region; see Figure 2.

It was proven in Popov and $\mathrm{He} \mathrm{B}^{22}$ for arbitrary axissymmetrical profiles that application of this rule reproduces the solution of Cattaneo/Mindlin and satisfies the Ciavarella-Jäger superposition principle. Below we will show that this procedure is valid also in the general case of arbitrary topographies using the equivalent profile obtained according to equation (5).

The simplest way to show this is to go from the Ciavarella-Jäger principle, which states that the tangential stress in a tangential contact with partial sliding can be expressed as

$$
\begin{aligned}
& \tau(\mathrm{x}, \mathrm{y})= \\
& \left\{\begin{array}{cc}
\mu \mathrm{p}_{1}(\mathrm{x}, \mathrm{y}) & \text { where sliding occurs } \\
\mu \cdot\left(\mathrm{p}_{\mathrm{l}}(\mathrm{x}, \mathrm{y})-\mathrm{p}_{\mathrm{c}}(\mathrm{x}, \mathrm{y})\right) & \text { where sticking occurs }
\end{array}\right.
\end{aligned}
$$

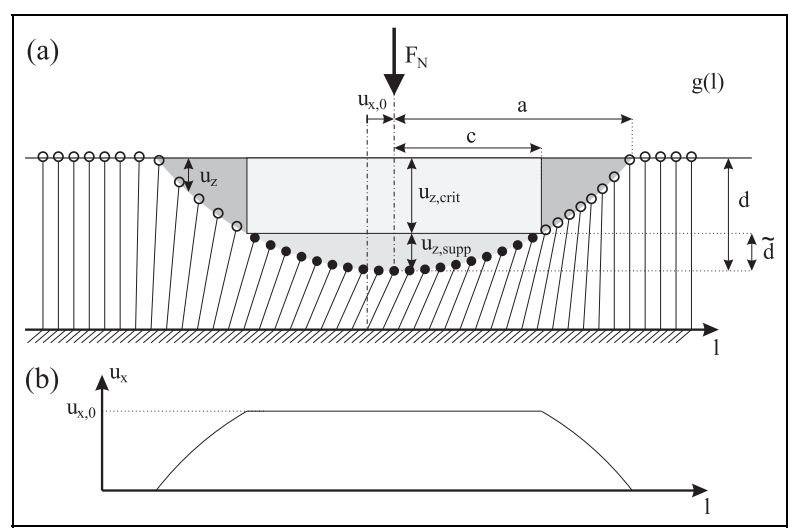

Figure 2. (a) MDR model for the normal and tangential contact. The transformed shape $g(l)$ is pressed into the foundation of independent springs, shown as lines. The deflection $\mathrm{u}_{\mathrm{z}}(\mathrm{I})$ depends on the global indentation depth $\mathrm{d}$ and the local value of $g(l)$. When a tangential motion is imposed, some springs stick (full circles) and some slip (open circles). (b) Local tangential deflection $u_{x}(l)$ for the above contact. Springs in the stick zone take the value of $u_{x}^{(0)}$. All other springs are in sliding state, and their tangential displacement is equal to $\mathrm{u}_{\mathrm{x}}(\mathrm{l})=\mathrm{u}_{\mathrm{z}}(\mathrm{I})\left(\mu \mathrm{E}^{*} / \mathrm{G}^{*}\right)$. where $\mathrm{p}_{1}(\mathrm{x}, \mathrm{y})$ is the pressure distribution in the current state which we can unambiguously characterize by the Holm radius 1 (hence the index " 1 "). $\mathrm{p}_{\mathrm{c}}(\mathrm{x}, \mathrm{y})$ is a corrective pressure distribution which is also a solution of the normal contact problem with the same geometry but a different indentation depth and thus corresponding to a different Holm radius, which we denote c. Integrating over the whole contact region, we get for the total tangential force

$$
\mathrm{F}_{\mathrm{x}}=\mu\left(\mathrm{F}_{\mathrm{N}}(l)-\mathrm{F}_{\mathrm{N}}(\mathrm{c})\right)
$$

where $\mathrm{F}_{\mathrm{N}}(1)$ is the normal contact force in the current state (corresponding to the Holm radius 1, and $\mathrm{F}_{\mathrm{N}}(\mathrm{c}$ ) is the normal contact force corresponding to the Holm radius $\mathrm{c}$ of the stick region.

In the equivalent MDR system, the very same principle is true. When a tangential deflection $\mathrm{u}_{\mathrm{x}}^{(0)}$ is imposed, all springs whose the tangential force is smaller than the normal force multiplied with the coefficient of friction will stick. The boundary of the stick region is given by the equality of the tangential force to the normal force times coefficient of friction: $G^{*} u_{x}^{(0)}=\mu E^{*} u_{z}(c)$. The tangential deflections outside the stick region are given by the condition $G^{*} u_{x}(x)=\mu E^{*} u_{z}(x)$. With $C_{m}=E^{*} / G^{*}$, the tangential force thus can be written as

$$
\begin{aligned}
F_{x} & =2 \int_{0}^{1} G^{*} u_{x}(x) d x=2 G^{*}\left(\int_{0}^{c} u_{x}(x) d x+\int_{c}^{1} u_{x}(x) d x\right) \\
& =2 G^{*}\left(\int_{0}^{c} \mu C_{m} u_{z}(c) d x+\int_{c}^{1} \mu C_{m} u_{z}(x) d x\right) \\
& =2 E^{*} \mu\left(\int_{0}^{c}\left(u_{z}(c)-u_{z}(x)\right) d x+\int_{0}^{1} u_{z}(x) d x\right) \\
& =\mu\left(F_{N}(l)-F_{N}(c)\right)
\end{aligned}
$$

which coincides with the Ciavarella and Jäger result, equation (17). A more detailed derivation of this result including the Ciavarella and Jäger superposition principle is given in the supplemental material to this article (available at: http://sdj.sagepub.com/).

\section{Examples of equivalent profiles}

In the previous section, we discussed how to find equivalent profiles for different original topographies. Here, we generate and discuss the equivalent profiles for selected cases which are not covered by the MDR transformation. All solutions are obtained using the boundary elements method as described in Pohrt and $\mathrm{Li}^{2}$ It iteratively finds a subset of discrete grid points in contact which satisfies the boundary conditions of having zero gap width inside and vanishing pressure outside the contact zone. Every subset of grid points defines an (not necessarily connected) area of contact from which one calculates the current Holm radius. In principle, one might as well record the normal force as 


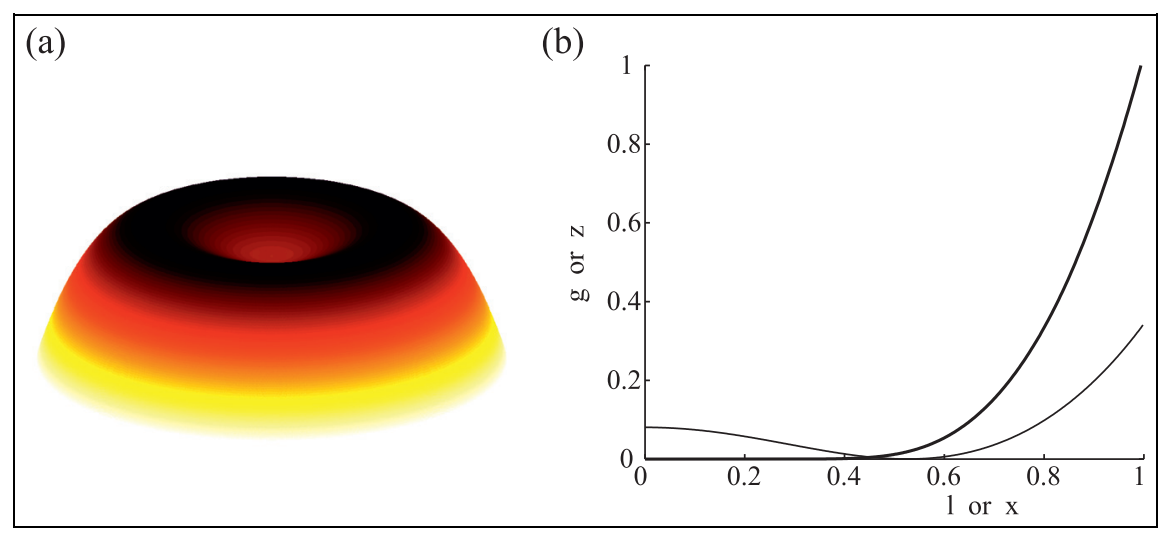

Figure 3. (a) Three-dimensional representation of an example of an axis-symmetric indenter shape. (b) Original profile as section of the body (thin line, negative sign) and equivalent MDR profile according to equation (5) (bold line).

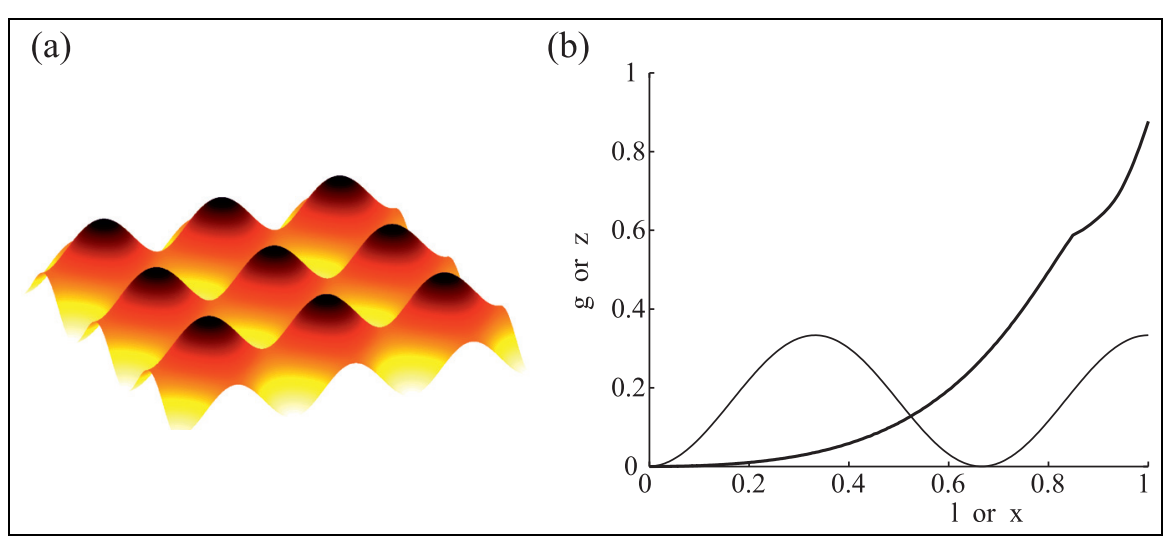

Figure 4. (a) Three-dimensional representation of an example of an ondulated shape. (b) Original profile as section of the body (thin line, negative sign) and equivalent MDR profile according to equation (5) (bold line).

the integral over the surface pressure and use its derivative with respect to $\mathrm{d}$. In the following figures, we show the indentation depth over the contact length (Holm radius) for different topographies as well as a plot of $-\mathrm{z}(\mathrm{x}, \mathrm{y})$ for $\mathrm{y}=0$ and $\mathrm{x}=[0 \ldots \mathrm{L} / 2]$ for comparison.

In Figure 3, we have chosen an axis-symmetric, shifted profile (see thin line in Figure 3(b)) which cannot be transformed using equation (14) because the resulting contact area at low loads is ring-shaped and thus not compact. In the equivalent profile, one can see that the Holm radius very quickly takes the value of flat torus radius ( 0.53 in the scaling). This is to be expected as the Holm radius is very much dominated by the maximum spatial spread of the contact region.

In Figure 4, we have generated a sinusoidal profile consisting of nine peaks that first enter into contact at isolated spots and later merge into a bigger contact area. This transition is indeed visible in the equivalent profile near $1=1$. The maximum Holm radius that can be reached is given by the square comprising all peaks $(1=1.1530 \mathrm{~L}$ where $\mathrm{L}=1$ is the edge of the square, not in plot).

The topography shown in Figure 5 is randomly rough and self-affine with Hurst exponent $\mathrm{H}=1$. As expected from equation (15), the resulting equivalent profile is approximately linear and only transitions to the saturation value of $1=\mathrm{L}$ at large $\mathrm{d}$.

Figure 6 shows a similar case where the roughness is applied onto a parabolic shape. There is a general semi-analytical solution available for particular cases of this scenario ${ }^{26}$ with fractal roughness in the absence of a long-wavelength cutoff in the power spectrum. However, in the current example, there is such a cutoff which makes the roughness appear nominally flat. Therefore, no analytical solution for the normal contact is available. The equivalent profile is instead obtained through direct simulation. The curve shows a transition to a $\mathrm{d} \propto \mathrm{l}^{2}$ dependency when the parabolic shape dominates the indentation behavior at high $\mathrm{d}$. Ultimately, a saturation in 1 is reached due to the finite shape.

\section{Numerical sample simulation}

In order to show the applicability of the proposed method, we now show an example for the tangential contact including loading history. The sample surface is the one depicted in Figure 6. All movements take 


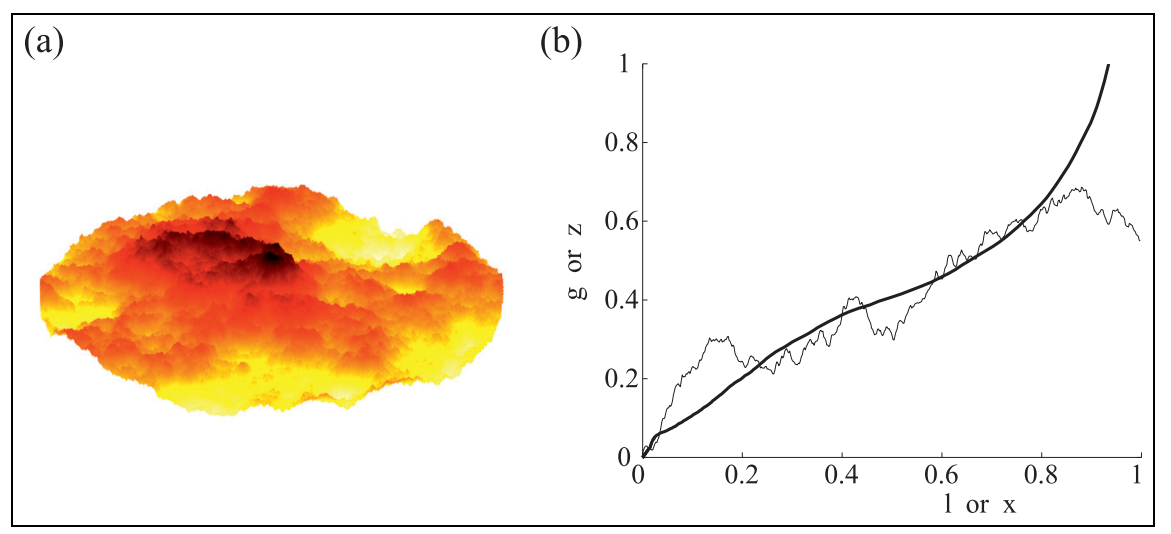

Figure 5. (a) Three-dimensional representation of an example of a randomly rough indenter shape. (b) Sample section of the body (thin line, negative sign) and equivalent MDR profile according to equation (5) (bold line).

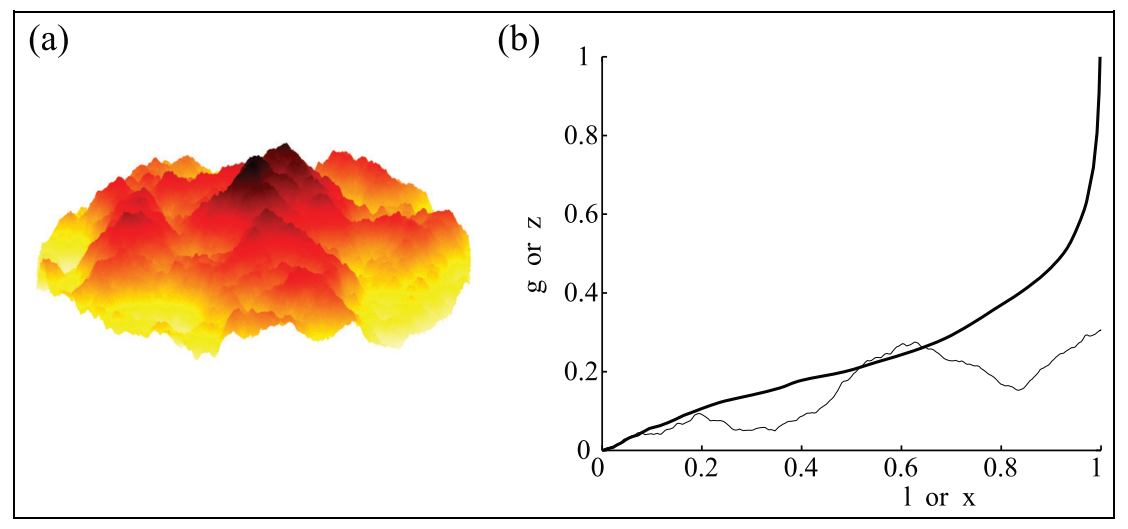

Figure 6. (a) Three-dimensional representation of an example of a rough parabolic indenter shape. (b) Original profile as sample section of the body (thin line, negative sign) and equivalent MDR profile according to equation (5) (bold line).

place at a constant indentation depth. The resulting contact area is constant and can be seen in the red and green spots in the right column of Figure 7.

This indented surface is subjected to an oscillating tangential movement with growing amplitude. We have simulated this case using boundary element methods with Cattaneo/Mindlin principle. For every time step, we have recorded the distribution of stick and slip area and the resulting tangential force, which is shown in Figure 8 .

We then used the equivalent profile in order to simulate the same tangential movements according to the rules of the MDR (see above). In Figure 7, the evolution of the spring deflections is easily interpreted. Because Coulomb friction is assumed, all deflections cannot exceed $\left|\mathrm{u}_{\mathrm{x}}\right| \leqslant \mathrm{u}_{\mathrm{z}} \mu \mathrm{C}_{\mathrm{m}}$. During tangential motion, the curve is simply shifted upward or downward, restricted by this boundary. The tangential force is obtained by evaluating the gray area. Points A and B show states shortly before and after the direction of motion is changed. In A, most of the contact zone slips. After the direction is changed (B), most points deliberately follow the external movement (they stick) with the exception of very lightly loaded points in the contact zone boundary. Please note that the same can be observed in the 1D model. Only a small fraction of the springs is quickly limited by the $\left|\mathrm{u}_{\mathrm{x}}\right| \leqslant \mathrm{u}_{\mathrm{z}} \mu \mathrm{C}_{\mathrm{m}}$ condition (red circle). In state $\mathrm{C}$, the curve of $\mathrm{u}_{\mathrm{x}}$ lowers again and conforms to $-\mathrm{u}_{\mathrm{z}} \mu \mathrm{C}_{\mathrm{m}}$ but still has the shape of the upper bound in all springs that are still in sticking state (left).

Figure 8 also shows the force-displacement dependency of the MDR calculation. Both curves are hardly distinguishable. However, the MDR procedure is dramatically simpler and requires only negligible computing time. The tangential force $F_{t}$ is normalized by the maximum value it can attain (coefficient of friction (COF) times normal force). The tangential bulk displacement $\mathrm{u}_{\mathrm{x}, 0}$ is normalized by its maximum value prior to macroscopic slip $\mathrm{u}_{\mathrm{x}, \max }$. For isotropic, elastic contact, this is given by $\mathrm{u}_{\mathrm{x}, \max }=\mathrm{d} \mu \mathrm{C}_{\mathrm{m}}$ (see Grzemba et al. ${ }^{27}$ for details).

\section{Discussion}

The MDR is an easy and effective method for treating various classes of contact problems by mapping them to the contact of a modified profile with a linear elastic (or viscoelastic) foundation. Often, it is erroneously 


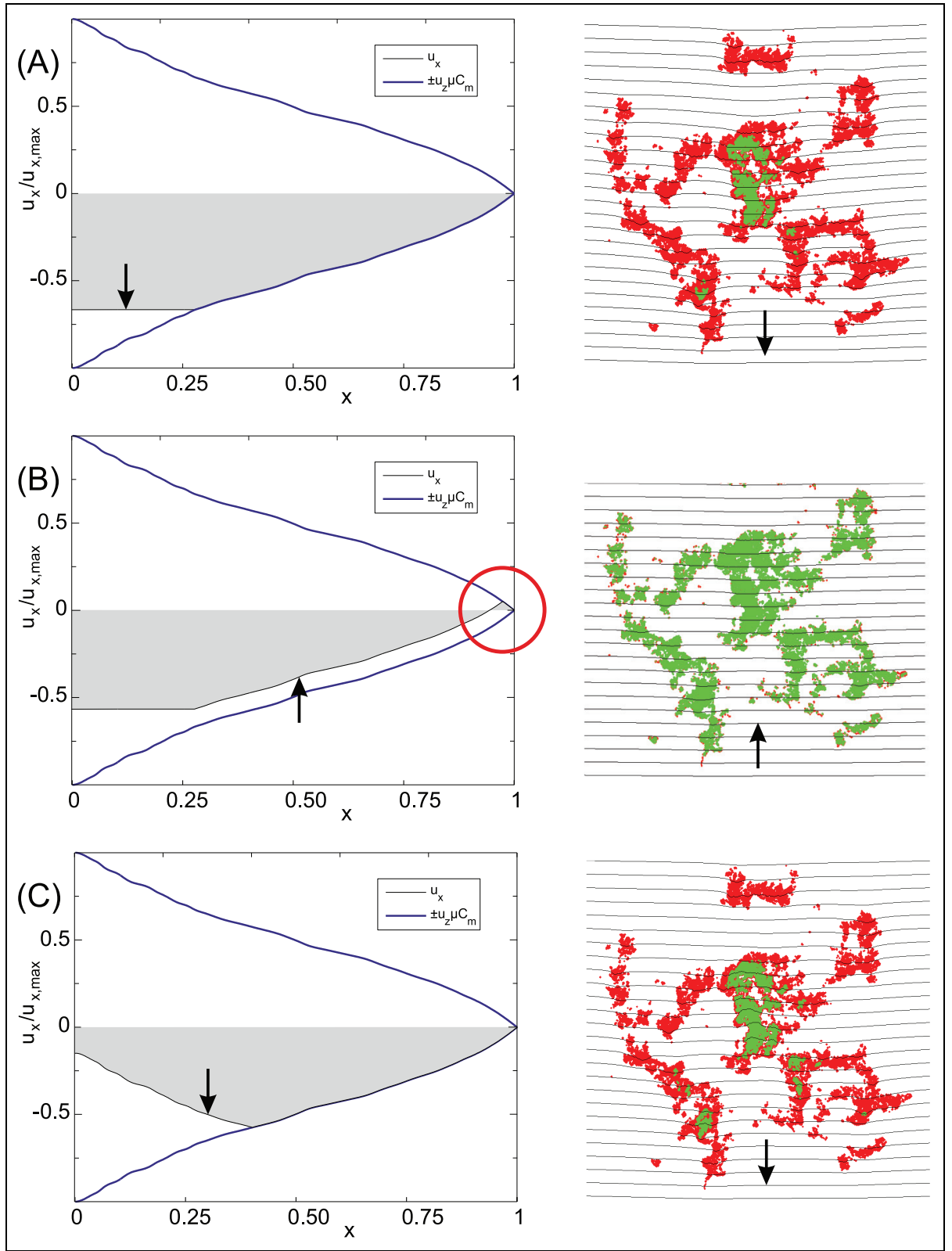

Figure 7. (Right column) Plot of the contact region of the surface shown in Figure 6. Green points are in sticking state, red points slide. We also show the tangential deflection of the outer surface by the horizontal lines. The states A-C correspond to the points marked in Figure 8. (Left column) Plot of the tangential deflection of the independent springs in the one-dimensional model.

believed that the MDR is only applicable to axissymmetric profiles. This is not correct. In this article, we have shown that the "equivalent 1D profile" does exist for absolutely every arbitrary surface shape. We have shown that this profile can be found from the known solution of the normal contact problem. The 1D profile is obtained directly by plotting the indentation depth over the contact length, when the dependency is known from analytical, numerical or experimental findings. Following Borri-Brunetto et al., ${ }^{20}$ the normal contact-based profile can then be used also for the simulation of the tangential contact problem with a constant coefficient of friction under arbitrary loading history. Here, we show a numerical example comparing
3D BEM and MDR results. Another application is the normal contact problem of any profile with an elastomer having arbitrary linear rheology. Also in this case, the MDR provides a powerful method which is easy to implement.

All the presented results are correct within the usual assumptions of linear contact mechanics (half-space approximation, geometrical linearity, Mindlin/ Cattaneo approximation and assumption of uncoupling of normal and tangential problems as well as neglecting the orthogonal slip).

It would be interesting to further investigate the applicability for wear prediction, currently shown only for axis-symmetric 3D shapes. ${ }^{28,29}$ 


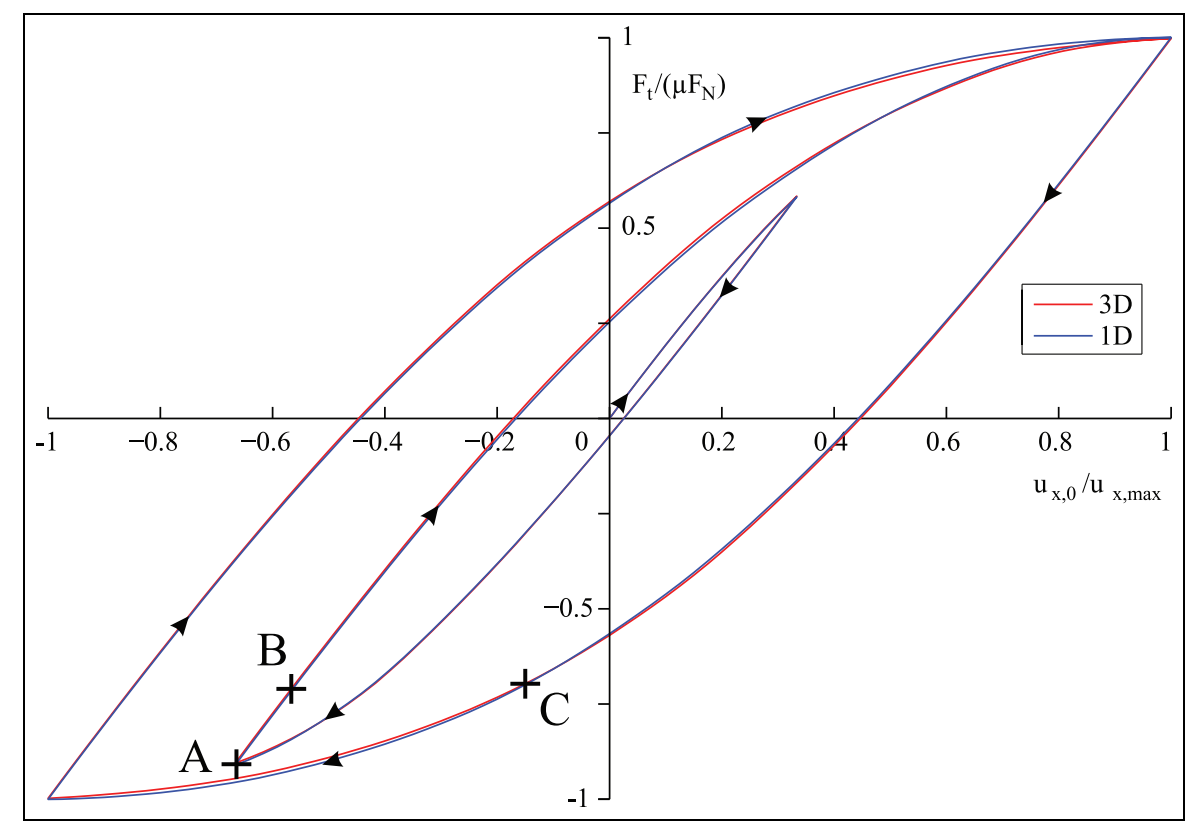

Figure 8. Tangential force-displacement curve for the tangential contact of the surface shown in Figure 6. The dependencies are generated using 3D boundary element method (red curve) and the ID method of dimensionality reduction based on the equivalent profile from Figure 6(b).

\section{Declaration of conflicting interests}

The author(s) declared no potential conflicts of interest with respect to the research, authorship and/or publication of this article.

\section{Funding}

The author(s) disclosed receipt of the following financial support for the research, authorship, and/or publication of this article: R. Pohrt received funding from project PO810/22-1 by Deutsche Forschungsgemeinschaft.

\section{References}

1. Hyun S, Pei L, Molinari JF, et al. Finite-element analysis of contact between elastic self-affine surfaces. Phys Rev E 2004; 70(2): 26117.

2. Pohrt R and Li Q.Complete boundary element formulation for normal and tangential contact problems. Phys Mesomech 2014; 17(4): 334-340.

3. Campana $\mathrm{C}$ and Müser MH.Contact mechanics of real versus randomly rough surfaces: a Green's function molecular dynamics study. Europhys Lett 2007; 77(3): 38005.

4. Yastrebov VA, Anciaux G and Molinari JF.From infinitesimal to full contact between rough surfaces: evolution of the contact area. Int J Solids Struct 2015; 52: 83-102.

5. Cattaneo C.Sul contatto di due corpi elastici: distribuzione locale degli sforzi. Atti Accad Naz Lin 1938; 27: 342-348, 434-436, 474-478.

6. Mindlin RD.Compliance of elastic bodies in contact. $J$ Appl Mech: T ASME 1949; 16: 259-268.

7. Mindlin RD and Deresiewicz H.Elastic spheres in contact under varying oblique forces. $J$ Appl Mech 1953; 20: $327-344$.
8. Jäger J.Axi-symmetric bodies of equal material in contact under torsion or shift. Arch Appl Mech 1995; 65: 478487.

9. Jäger J.Half-planes without coupling under contact loading. Arch Appl Mech 1997; 67(4): 247-259.

10. Ciavarella M.Tangential loading of general threedimensional contacts. $J$ Appl Mech 1998; 65: 998-1003.

11. Ciavarella M.The generalized Cattaneo partial slip plane contact problem. I-theory. Int J Solids Struct 1998; 35(18): 2349-2362.

12. Johnson KL.Surface interaction between elastically loaded bodies under tangential forces. P Roy Soc Lond A Mat 1955; 230: 531-548.

13. Munisamy RL, Hills DA and Nowell D.Static axisymmetric Hertzian contacts subject to shearing forces. $J$ Appl Mech: T ASME 1994; 61: 278-283.

14. Dini D and Hills DA.Frictional energy dissipation in a rough Hertzian contact. J Tribol: T ASME 2009; 131(2): 21401.

15. Barber JR, Davies M and Hills DA.Frictional elastic contact with periodic loading. Int J Solids Struct 2011; 48(13): 2041-2047.

16. Putignano $C$, Ciavarella $M$ and Barber JR.Frictional energy dissipation in contact of nominally flat rough surfaces under harmonically varying loads. $J$ Mech Phys Solids 2011; 59(12): 2442-2454.

17. Galin LA. Contact problems in the theory of elasticity. Raleigh, NC: North Carolina State College, 1961 (This book is an English translation of the Russian original of 1953: Л.А. Галин, Контактные задачи теории упругости. M., 1953).

18. Sneddon IN.The relation between load and penetration in the axisymmetric Boussinesq problem for a punch of arbitrary profile. Int J Eng Sci 1965; 3: 47-57.

19. Aleshin V, Bou Matar O and Van Den Abeele K.Method of memory diagrams for mechanical frictional contacts 
subject to arbitrary 2D loading. Int $J$ Solids Struct 2015; 60: 84-95.

20. Borri-Brunetto $\mathbf{M}$, Carpinteri A, Invernizzi $\mathbf{S}$, et al. Micro-slip of rough surfaces under cyclic tangential loading. In: Wriggers P and Nackenhorst U (eds) Analysis and simulation of contact problems. Berlin, Heidelberg: Springer, 2006, pp.333-340.

21. Popov VL.Method of reduction of dimensionality in contact and friction mechanics: a linkage between micro and macro scales. Friction 2013; 1(1): 41-62.

22. Popov VL and Heß M.Method of dimensionality reduction in contact mechanics and friction. Berlin, Heidelberg: Springer, 2015.

23. $\mathrm{Heß} \mathrm{M.Über} \mathrm{die} \mathrm{exakte} \mathrm{Abbildung} \mathrm{ausgewählter} \mathrm{dreidi-}$ mensionaler Kontakte auf Systeme mit niedrigerer räumlicher Dimension. Göttingen: Cuvillier Verlag, 2011.

24. Radok JRM. Visco-elastic stress analysis. $Q$ Appl Math 1957; 15: 198-202.
25. Pohrt R, Popov VL and Filippov AE. Normal contact stiffness of elastic solids with fractal rough surfaces for one- and three-dimensional systems. Phys Rev E 2012; 86: 026710

26. Pohrt R and Popov VL.Contact mechanics of rough spheres: crossover from fractal to Hertzian Behavior. Adv Tribol 2013; 2013: 974178.

27. Grzemba B, Pohrt R, Teidelt E, et al. Maximum microslip in tangential contact of randomly rough self-affine surfaces. Wear 2014; 309(1): 256-258.

28. Dimaki AV, Dmitriev AI, Chai YS, et al. Rapid simulation procedure for fretting wear on the basis of the method of dimensionality reduction. Int $J$ Solids Struct 2014; 51(25): 4215-4220.

29. Dimaki AV, Dmitriev AI, Menga N, et al. Fast highresolution simulation of the gross slip wear of axially symmetric contacts. Tribol T. Epub ahead of print 6 July 2015. DOI: 10.1080/10402004.2015.1065529. 